\title{
Successful Completion of the Top-off Upgrade of the Advanced Light Source
}

\author{
C. Steier, B. Bailey, K. Baptiste, W. Barry, A. Biocca, W. Byrne, P. Casey, M. Chin, \\ R. Donahue, R. Duarte, M. Fahmie, B. Gath, S. Jacobson, J. Julian, J.Y. Jung, M. \\ Kritscher, S. Kwiatkowski, S. Marks, P. McKean, R. Mueller, H. Nishimura, J. \\ ONeill, G. Portmann, S. Prestemon, D. Robin, S. Rossi, F. Sannibale, T. Scarvie, R. \\ Schlueter, D. Shuman, A. Smith-Baumann, G. Stover, C. Timossi, W. Wan, T. \\ Warwick, R. Wells, J. Weber and E. Williams
}

LBNL, Berkeley, CA 94720, USA

\begin{abstract}
.
An upgrade of the Advanced Light Source to enable top-off operation has been completed during the last four years. The final work centered around radiation safety aspects, culminating in a systematic proof that top-off operation is equally safe as decaying beam operation. Commissioning and transition to full user operations happened in late 2008 and early 2009. Top-off operation at the ALS provides a very large increase in time-averaged brightness (by about a factor of 10) as well as improvements in beam stability. The following sections provide an overview of the radiation safety rationale, commissioning results, as well as experience in user operations.
\end{abstract}

Keywords: Synchrotron Light Source, Storage Ring, Injection, Brightness PACS: $29.20 . \mathrm{db}, 29.27 . \mathrm{Ac}$, 41.75.Fr, 41.85.Ar, 52.59.-f

\section{INTRODUCTION}

The Advanced Light Source (ALS) at Lawrence Berkeley National Laboratory is one of the earliest 3rd generation light sources. It continues to generate forefront science with more than 2000 users every year. Many upgrades of the ALS have been implemented over the years, the most recent one being a conversion to enable top-off operation.

Top-off operation [1] is now widely used and provides two main benefits. Because of the constant beam current, it can improve the overall stability. It also allows to operate with smaller beam lifetimes (within limits imposed by radiation safety, injector abilities and the frequency of injection users are willing to tolerate). In a low energy light source, this allows to significantly reduce the vertical emittance and therefore increase the brightness. With the increase in average current as well as the reduction in vertical emittance in the ALS there is almost one order of magnitude improvement in brightness above $2 \mathrm{keV}$. With those parameters the ALS achieves similar brightness in the soft x-ray range around $1 \mathrm{keV}$ as the newest light sources (e.g. Soleil, Diamond). Additional upgrades that have just been started will allow significant further improvements.

\section{INJECTOR UPGRADE}

The top-off upgrade started in 2004 with a conceptual design phase [2]. The conceptual design report [3] was finished by the end of 2004. The project started in earnest in early 2005, when the funding for the main part of the upgrade was received. Detailed design of all long lead items followed. The main areas of work were:

- New power supplies, RF transmitter, pulsed magnet upgrades for booster and transfer lines for full energy injection (upgrade from 1.5 to $1.95 \mathrm{GeV}$ booster energy).

\footnotetext{
${ }^{1}$ Work supported by the U.S. Department of Energy under Contract No. DE-AC02-05CH11231

2 CSteier@lbl.gov
} 
- Radiation Safety studies to demonstrate that top-off operation of the ALS is safe and associated system installation (radiation monitors, collimators, interlocks, apertures, shielding).

- Upgrades to timing systems, controls, diagnostics and new top-off compatible bunch cleaning system.

Most hardware components were installed in late 2006 and successfully commissioned. After resolving several challenges with the new booster power supplies, which initially prevented us from reaching $1.9 \mathrm{GeV}$, we finally achieved full energy injection into the storage ring later in 2007. In parallel experimental radiation safety studies were conducted and a complete rationale was developed to prove that top-off operation of the ALS is safe. Some results will be explained in the following section.

\section{RADIATION SAFETY}

Radiation safety was the most important and most challenging area of the top-off upgrade [4]. The radiation dose rate of even one bunch of electrons propagating down a synchrotron light beamline could be dangerously high, therefore this case had to be excluded with absolute certainty. We closely considered the radiation safety approaches at facilities which already use top-off injection, namely APS, ESRF, SLS, and Spring-8. A comprehensive explanation of the ALS radiation safety approach can be found in [5]

Since it is impossible to demonstrate experimentally that the accident scenario of the injected beam traveling down a user beamline cannot happen, we followed the approach of other facilities to demonstrate the safety of top-off injection with tracking studies. For the tracking studies, a new tracking routine was implemented, since no codes were available that included all necessary effects [6]. Challenges in the tracking are the very large oscillation amplitudes of the particles as well as the extremely large number of parameter combinations one has to study.

As a result of the tracking studies we came up with a detailed list of mitigation measures. They consist of several interlock systems, that only allow injection with beamline shutters open, if there is stored beam in the storage ring, the beam energy of the booster is very close to the storage ring, several storage ring magnets are close enough to their nominal settings, as well as regular alignment surveys of important apertures in the storage ring and beamline frontends. We also installed new apertures in several beamlines as well as interlocked radiation monitors on user beamlines. In parallel we went through the elaborate process to receive regulatory approval, which was received in October 2008.

\section{COMMISSIONING}

The commissioning of top-off happened in many stages. It started with early tests including users during the design stages to establish requirements for the gating system and minimization of injection transients. Later it included injection tests on one user beamline with open safety shutter. The beamline was modified with substantial amounts of additional lead shielding for this test. This allowed early study of routine radiation backgrounds during injection with shutters open.

The next stage was the most time intensive one, it included the full recommissioning of the injector and transfer lines, after they were upgraded for full energy injection. Because of problems with some of the new power supplies, this stage also was the most challenging one and required quick implementation of fallback control equipment and software to allow the use of the new powersupplies. The final stage of actual commissioning of the top-off interlocks as well as the initial radiation measurements with all beamline apertures open went very well. No surprises were found and all radiation dose rates in routine operation as well as in ppor injection efficiency conditions are low. During this final stage we also demonstrated the design current stability $( \pm 0.15 \%)$, increase in current to $500 \mathrm{~mA}$, as well as reduction in vertical emittance for users (which was previously demonstrated during dedicated machine shifts).

\section{USER OPERATIONS}

The switchover to user operations worked extremely well. It was accomplished in early February 2009, once the qualification of all interlock systems as well as the final beamline modifications with apertures were completed. Fig. 1 shows the comparison of the $12 \mathrm{~h}$ ALS status displays for decaying beam operations and top-off mode. Currently we inject about $1.2 \mathrm{~mA}$ every $40 \mathrm{~s}$, i.e. the beam current is stable to about $0.25 \%$ (peak-to-peak). 

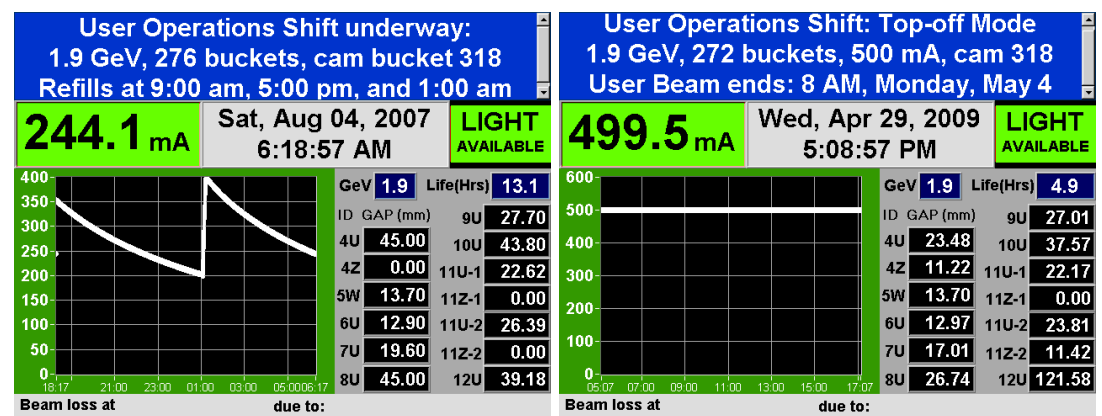

FIGURE 1. Snapshots of the 12 hour ALS status display in decaying beam operation as well as top-off. Note the difference in vertical scale (400 vs. $600 \mathrm{~mA})$.

The overall reliability of the ALS during the initial operation in top-off mode has been good. In fact, the reliability has been substantially higher in the first seven months of operation with top-off than it was in the six months preceding those. The increase in current to $500 \mathrm{~mA}$ has not created any significant problems. We have also succeeded in reducing the vertical emittance by about a factor of 2.5 to $50 \mathrm{pm}$ in user operations, which was made possible because the lower Touschek lifetime resulting from this can be tolerated in top-off. A further reduction to about $30 \mathrm{pm}$ is planned for the next few months, which will mean that all soft x-ray (undulator) beamlines at the ALS will be diffraction limited in the vertical plane. User response has been extremely positive and only a small fraction of beamlines needs to make use of the provided injection gating signal.

\section{Stability}

The second main goal for top-off operations, besides increasing the brightness, is improving the overall beam stability. This achieved by reducing thermal effects, as well as eliminating current dependent errors in beam position monitors. A measurable improvement was achieved as can be seen in Fig. 2. The main improvement is in the vertical plane is achieved mostly by the elimination the current dependence of the BPM system. Now the mid term stability over one day is approximately 1 micron.
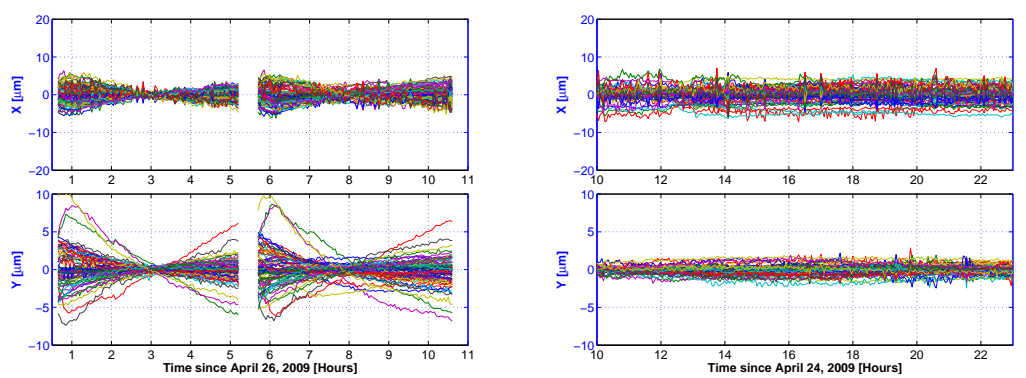

FIGURE 2. Medium term (10-20 h) orbit stability for top-off mode (right) compared to decaying beam operation (left).

In addition, the effect of constant heatload on user beamline optics has been very beneficial. Many of the beamlines have reported significant improvements in the long term stability of their beamline optics.

\section{Twobunch Operation}

The ALS operates for about 3-4 weeks a year in two bunch operation. In this mode, contamination in all other buckets needs to be actively cleaned. The method used to do this historically at the ALS made use of the tune shift with current. However, it was incompatible with top-off. Therefore a new system was installed which mixes a vertical excitation signal used for cleaning with a square wave that has zero crossings at the location of the bunches one wants to keep. This method has been used before in booster synchrotrons [7, 8]. However, for the case of the ALS, because 
of the low beam energy and short Touschek lifetime, this would not be sufficient. To also clean contamination resulting from Touschek induced diffusion in the storage ring, the new cleaning system at the ALS is implemented on the storage ring.

There was a first two-bunch user run in top-off in March and the new cleaning system performed extremely well. It is used for about $300 \mathrm{~ms}$ after every injection and was transparent for users. They did not use the gating signal provided while the bunch cleaning system was on. Overall the time average current during two-bunch top-off operations was almost a factor of 3 higher (compare Fig. 3), the emittance a factor of 2 smaller and the bunch purity substantially better.
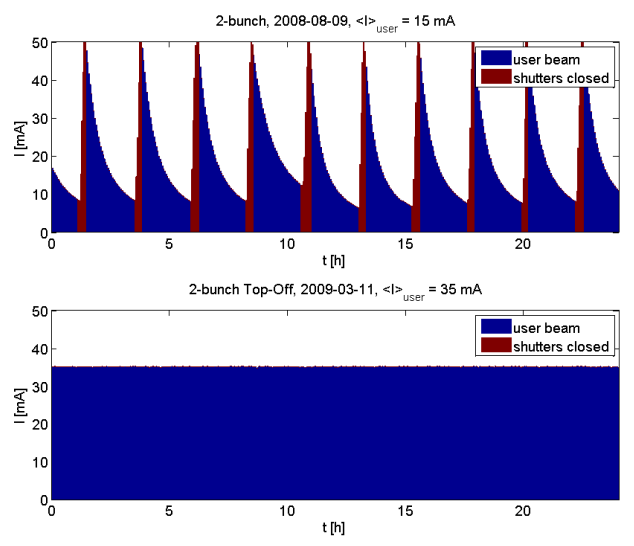

FIGURE 3. One day current history of two bunch operation in decaying beam mode and top-off. The average current is almost tripled (and shutters always stay open).

\section{CONCLUSIONS}

The upgrade of the ALS for top-off operation has been successfully completed. The project took about 4 years and had to deal with some setbacks along the way. However, the final commissioning worked very smoothly including all new radiation safety systems. Dose rates in routine operation as well as for worst case injection conditions are low. Only minimal teething pains were encountered in over seven months of user operations so far, and design parameters (including in two bunch operation) were reached quickly. The reaction by the ALS user community was extremely positive.

\section{ACKNOWLEDGMENTS}

We would like to thank many colleagues from other light sources, who have shared their experience in implementing top-off operation with the authors. We would like to particularly thank Louis Emery, Michael Borland, Laurent Farvacque, Michael Boege, Lenny Rivkin, and Peter Kuske, as well as the SPEAR accelerator physics group.

\section{REFERENCES}

1. L. Emery, M. Borland, Proc. PAC99, New York (1999) 200

2. D. Robin, et al., Proc. EPAC04, Lucerne (2004) 2442

3. C. Steier, D. Robin, CDR of the Top-Off Upgrade of the ALS at LBNL, LBNL/Pub-5506 (2004)

4. C. Steier, et al., Status of the Top-Off Upgrade, Proc. EPAC06, Edinburgh (2006)

5. H. Nishimura, et al., Advanced light source's approach to ensure conditions for safe top-off operation, NIM A, 608, 1, (2009) 2

6. H. Nisimura, et al., ALS Top-off Simulation Studies for Radiation Safety, Proc. EPAC08, Genova (2008)

7. H. Suzuki, et al., NIM A 444 (2000) 515

8. N. Sereno, Proc. PAC99, New York, (1999) 2322. 